\title{
Development of Marathi Version Cross-Culture Adaptation, Reliability and Validity of Shoulder and Disability Index
}

\author{
T. Poovishnu Devi ${ }^{1}$, Sapnashamrao Khot ${ }^{2}$ \\ ${ }^{1}$ Associate Professor, Krishna College of Physiotherapy, KIMSDU, Karad, Maharastra, India \\ ${ }^{2}$ Clinical Physiotherapist, Qi Spine Clinic, Ghatkopar Branch, Mumbai, Maharastra, India
}

\begin{abstract}
The prevalence of shoulder pain is about 6-37\%. Shoulder pain and disability index (SPADI) is most frequently used tools to evaluate disability in shoulder pain patients. Its psychometric properties are highly reliable. Currently no validated Marathi version is available. Objective is to translate, culturally adapt and validate SPADI version. The psychometric properties are evaluated by administering to questionnaire to 120 subjects with shoulder pain. Test retest reliability was estimated by test retest and internal consistency assessment. Test retest reliability of SPADI questionnaire was calculated using intra class correlation co efficient. The results shows excellent test retest reliability (ICCC.97) and concluded that spadi shows excellent test retest reliability.
\end{abstract}

Keywords: SPADI, shoulder pain, cross culture adaptation, reliability, validity

\section{Introduction}

The shoulder dysfunction is the most common cause of musculoskeletal pain secondary to back pain and $6-37 \%$ of the general population reported prevalence of shoulder pain from 1 month to 1 year of duration.[1] Patient oriented outcome tools i.e questionnaire, it offers abundant information to the therapist regarding patients level of function and also helps in clinical decision making process. [2]Several self report measures have been developed in English for measuring shoulders functionality and has been encouraged the translation cross-cultural adaptation and validation of these tools rather than creating a new instruments which avoids indiscriminate proliferation of assessment tools.[3]A shoulder pain and disability index (SPADI) was developed to measure the pain and disability associated with shoulder pathology.[4]

SPADI is a self administered questionnaire that consists of two dimensions, one for pain consists of 5 items regarding the severity of an individual's pain, another for functional activities consists of 8 items designed to measure the degree of difficulty an individual has with various activities of daily living that require upper extremity use. There are two versions of the SPADI; the original version has each item scored on a visual analogue scale (VAS) and a second version has items scored on a numerical rating scale (NRS). The latter version was developed to make the tool easier to administer and score. Both versions take less than five minutes to complete. SPADI takes 5-10 minutes for a patient to complete and is only reliable and valid region specific measure for the shoulder.[5] Besides the Original English version, the Shoulder Pain And Disability Index has been translated and cross culturally adapted in Danish [6], Hindi [7], Slovene [8], Spanish [9], Turkish.[10]

Maharashtra is one of the major states of north India. Maharashtra is second populous states in India According to Population census of 2001, the Population of Maharashtra was 112 million. The Population of Maharashtra increased by $17.20 \%$ as compared to last census of India in 1991 . Maharashtra is one of the top states in terms of literacy rate in India. Mumbai is the top city with over 18 million populations in Maharashtra. $65 \%$ of the population is speaking Marathi language. This shows how much the translation of SPADI into Marathi language is important to understand their problems without the barrier of language. Till now no version of SPADI in Marathi language is available. As Shoulder pain and disability index is self reported questionnaire and the population taken in this study from Maharashtra state is having mother tongue marathi which leads to translate the questionnaire in marathi language so it can reduce the barrier of language and gives valid and reliable data or information for research and clinical purpose.

\section{Need of the Study}

SPADI has become one of the principle outcome measurement used in management of shoulder problems. Thus lack of a standard reliable and valid instrument in Marathi language measuring disability of shoulder pain, leads to prepare and test the reliability and validity of a Marathi version of SPADI among Marathi speaking Indian patients with shoulder pain.

Aim: To adapt the SPADI into Marathi version following a systematic standardized process.

Objective: To translate a version of the SPADI into Marathi and evaluate its reliability.

\section{Literature Survey}

Christiansen DH et al.(2013): Conducted study with an objective of cross culturally adapt the Danish version of the Shoulder pain and disability index and to evaluate its measurement properties in terms of reliability and known group validity. A total of 65 patients with a variety of 


\section{International Journal of Science and Research (IJSR) \\ ISSN (Online): 2319-7064}

Index Copernicus Value (2015): 78.96 | Impact Factor (2015): 6.391

shoulder diagnoses were included in the study. The minimal detectable change was estimated to 19.4 points, and the testretest reliability measured by intraclass correlation coefficient was 0.88 (95\% confidence interval 0.83 to 0.94 ). Internal consistency measured by Cronbach's alpha was 0.94 .This study concluded that The Shoulder Pain and Disability Index provides a sensitive and reliable tool to assess pain and disability in Danish -speaking orthopaedic shoulder patients.

Sharma neha et al. (2011): Conducted study with an objective on Translation and Adaptation of Shoulder pain and disability index in Hindi. Minimal discrepancies were found during the process with respect to the concept and construct which were taken care with successive forward and backward translations. The translation produced using the AAOS(American Academic Of Orthopedic Of Surgeon) model was pretested on selected subjects in order to achieve a promising Hindi version of SPADI. test-retest reliability measured by ICC and internal consistency by Cronbach's alpha. Study shows that SPADI-Hindi is a well translated and adapted instrument for use in patients with musculoskeletal shoulder pathologies

Jaquelins Martin et al.(2010): conducted study with an objective of To translate and culturally adapt the Shoulder Pain and Disability Index(SPADI) into Brazilian-Portuguese and to assess its reliability. The data from the translation were analyzed descriptively, the test-re-testreliability by Intraclass Correlation Coefficient (ICC) and the internal consistency by Cronbach's Alpha. A total of 32 patients with shoulder dysfunction were included in the study. The testretest reliability ranged from 0.90 to 0.94 and the internal consistency ranged from 0.87 to 0.89 . This study concluded that after the translation and cultural adaptation, it was obtained a reliable version of SPADI-Brazil.

Bicer A et al.(2010): Conducted study with an objective of To test the Convergent validity and reliability of Shoulder Pain And Disability Index(SPADI) in Turkish women. A total of 101 female patients were enrolled in the study. The SPADI and HAQ (Health Assessment Questionnaire) were completed by all the participants. Test-retest reliability measured by ICC and internal consistency by Cronbach's alpha. Value for the SPADI was found to be 0.94 .Test retest reliability of the SPADI was found to be very high (0.92).The correlation coefficient for convergent validity of the SPADI 0.65 and 0.67 for the VAS and HAQ over all scores. This study concluded that the SPADI is a valid and reliable instrument to access the shoulder's pain in Turkish females was developed.

Jamnik et al.(2008): Conducted study with an objective of To translate the Shoulder Pain And Disability Index(SPADI) into Slovene language and assess its reliability and validity. A total of 52 participants with shoulder problems participated in the study. Test-retest reliability and internal consistency of SPADI were tested by intraclass correlation coefficient and Cronbach's [alpha], respectively. Construct validity was evaluated using principal components analysis. The instrument demonstrated high test-retest reliability (intraclass correlation coefficient SPADI 0.94, pain subscale 0.89 and disability subscale 0.95 ) and high to moderate internal consistency (Cronbach's alpha SPADI 0.92, pain subscale 0.78 and disability subscale 0.90). This study concluded that The Slovene version of the SPADI is a valid and reliable instrument.

F. Angst et al.(2007): Conducted study with an objective of To cross-culturally adapt the Shoulder Pain and Disability Index (SPADI) from English into German, and to test the reliability and validity of the German version. One hundred and eighteen patients who had undergone shoulder arthroplasty, on average $4 \mathrm{yr}$ previously completed a questionnaire booklet containing the German SPADI, the Short Form 36 (SF-36), the Disability of the Arm, Shoulder and Hand (DASH) questionnaire, and the American Shoulder and Elbow Surgeons (ASES) questionnaire for the shoulder to assess SPADI's construct validity. Test-retest reliability and internal consistency measured by ICC and Cronbach's alpha. The intraclass correlation coefficients for the individual items of the SPADI were between 0.68 and 0.89 , and that for the SPADI total score was 0.94. The SPADI total score showed a correlation of $0.61-0.69$ with the SF-36 physical scales, of 0.88 with the DASH and of 0.92 with the ASES. This study concluded that the SPADI is a valid and reliable instrument.

Jose Alvarez -Nemegyei et al.(2005): Conducted study with an objective of To develop and test the consistency of the Spanish translation of the Netherlands version of the SDQ(NSDQ). Both NSDQ and S-SDQ were administered to 30 bilingual (English/ Spanish) subjects. Test-retest reliability and internal consistency measured by ICC and Cronbach's alpha. In the bilingual subjects, the intraclass correlation coefficient between NSDQ and S-SDQ scores was 0.991 (95\% confidence interval, 0.981-0.995). In the monolingual Spanish speaking patients with shoulder pain the intraclass correlation coefficient between the 2 S-SDQ scores was 0.99 ( 95\% confidence interval, 0.997-0.999). The Cronbach's alpha coefficient for the S-SDQ was 0.99 .This study concluded that it is highly consistent Spanish version of the NSDQ, the S-SDQ

\section{Methods}

Source of data: Physiotherapy sectors of health centres and hospitals.

Sampling Design: Observational study to validate a scale.

Sampling: purposive sampling

\section{Inclusion Criteria}

1) Shoulder rotator cuff injury

2) Shoulder impingement syndrome.

3) Shoulder pain.

4) Fractures of the shoulder bones

5) Individual who can read and understand marathi language

6) Age group: $20 \mathrm{yrs}-70 \mathrm{yr}$

Exclusion criteria

1) Neurological conditions

2) Psychiatric conditions [9]

\section{Volume 6 Issue 7, July 2017 www.ijsr.net}




\section{International Journal of Science and Research (IJSR) \\ ISSN (Online): 2319-7064}

Index Copernicus Value (2015): 78.96 | Impact Factor (2015): 6.391

Materials used: Shoulder pain and disability indexMarathi version questionnaire.

\section{Statistical analysis: Intra class coefficient}

Tools used for data collection: SPADI questionnaire sheet, pen, pad, calculator

\section{Methods for Data Collection}

\section{Cross-culture adaptation process:}

In order to ensure the quality of adaptation this study followed the essential steps recommended. These steps are:

Step 1: Initial translation in to Marathi language/Forward translation:

Initial translation of Shoulder pain and disability index were done into the marathi language from original English version of Shoulder pain and disability index Forward translations were performed by two translators who knew both English and Marathi languages. The first translator was a translator of medical background who was aware of the concept being measured and the second translator was a translator of non medical background and was blind to the concept being measured.[10]The two translated version was compared and analyzed until a consensus regarding translation synthesis and the final synthesis version has formed.

\section{Back-translation:}

The final synthesis of Marathi version was back translated into English version by two different professional translators and who did not participate in the previous phase (forwardtranslation). These translators neither aware or nor informed about the concept explored in the questionnaire.From these two translated English version final synthesis version was formed.Final synthesized English version was compared with the original English version.Final Marathi version of Shoulder pain and disability index was then jointly reviewed by a bilingual team, including the four translators, one public health physician and three physiotherapists, to assess the necessity of performing a culture-adaptation and fine tune it for use among the marathi patients and final stage of adaptation was performed as process test of pre final version. With this back translation CONTENT VALIDITY of the questionnaire was warranted.[11]

\section{Step 2.Reviewers' Committee}

Synthesized marathi version of SPADI was then jointly reviewed by a bilingual team which includes four translators, one public health physician and three physiotherapists, to assess the necessity of performing a culture-adaptation and fine tune it for use among the Marathi knowing patients. Final stage of adaptation was performed and pre final version has been made. [11]

\section{Step 3}

Pilot study: To check for any errors in interpretation, a pilot study was done. It was carried out with a sample of 10 Shoulder pain patients according to inclusion criteria. They were asked to fill the questionnaire, and give the feedback and comments regarding the each questions and any difficulty in understanding it. On the basis of their reviews the final version of questionnaire was developed which would be approved by the committee.

\section{Step-4:}

Implementation of Marathi version of ODI A cross-culture adaptation was done by applying the Marathi version of SPADI on 120.patients with Shoulder Pain. SPADI was given twice in 24 hour interval to estimate test-retest reliability of it

\section{Evaluation of psychometric properties:}

Reliability: Would be estimate through stability and internal consistency assessment. Patients with Shoulder pain was tested twice in a 24 hours interval under similar condition.

Validity: Content validity.[12]

\section{Translation process}

\section{Results \& Discussion}

Table 1: Age Groups

\begin{tabular}{|c|c|c|}
\hline Age & frequency & Percent \\
\hline $21-30$ & 28 & 23.3 \\
\hline $31-40$ & 33 & 27.5 \\
\hline $41-50$ & 21 & 17.5 \\
\hline $51-60$ & 14 & 11.7 \\
\hline Above 60 & 24 & 20.0 \\
\hline Total & 120 & 100.0 \\
\hline
\end{tabular}

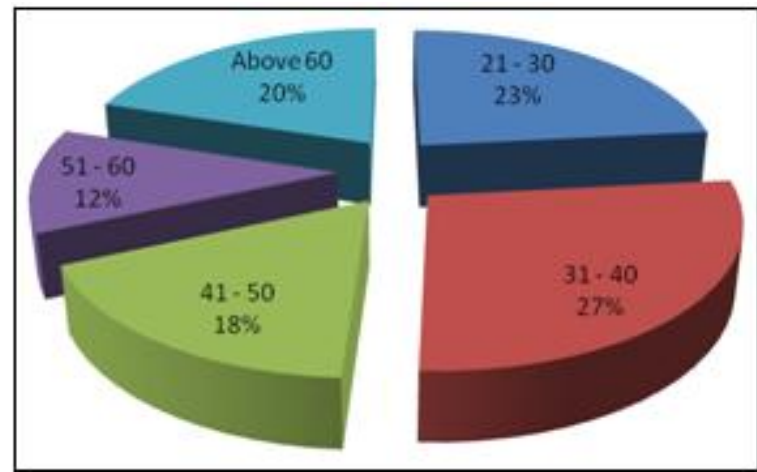

Graph 1: Age group

Interpretation: no of different age groups participated in the study.

Table 2: Gender

\begin{tabular}{|c|c|c|}
\hline Sex & Frequency & Percent \\
\hline Female & 69 & 57.5 \\
\hline Male & 51 & 42.5 \\
\hline Total & 120 & 100.0 \\
\hline
\end{tabular}

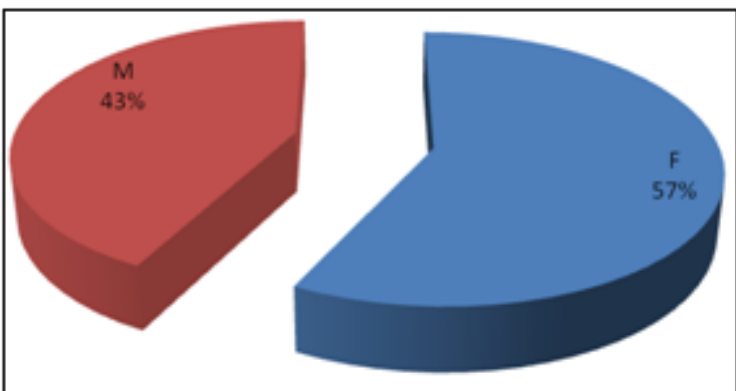

Graph 2: Gender

\section{Volume 6 Issue 7, July 2017 www.ijsr.net}




\section{International Journal of Science and Research (IJSR) \\ ISSN (Online): 2319-7064 \\ Index Copernicus Value (2015): 78.96 | Impact Factor (2015): 6.391}

Interpretation: No of Females and Males Participated In The Study

Table 3: Mean, $\mathrm{SD}$, ' $\mathrm{t}$ ' and $\mathrm{P}$ value

\begin{tabular}{|c|c|c|c|c|c|c|c|}
\hline Gender & $\mathrm{N}$ & Mean & SD & $\begin{array}{c}\text { Mean } \\
\text { diff }\end{array}$ & $\begin{array}{c}\text { SD of } \\
\text { diff }\end{array}$ & $\begin{array}{c}\text { ' } \mathrm{t} \text { ' } \\
\text { value }\end{array}$ & $P$ value \\
\hline $\begin{array}{c}\text { F day I } \\
\text { day II }\end{array}$ & & & & & & 90 & \\
\hline $\begin{array}{c}\text { M day I } \\
\text { day II }\end{array}$ & $\begin{array}{l}51 \\
51\end{array}$ & $\begin{array}{l}69.90 \\
69.90\end{array}$ & $\begin{array}{l}22.71 \\
21.98\end{array}$ & .008 & 6.735 & .008 & Ns.993 \\
\hline
\end{tabular}

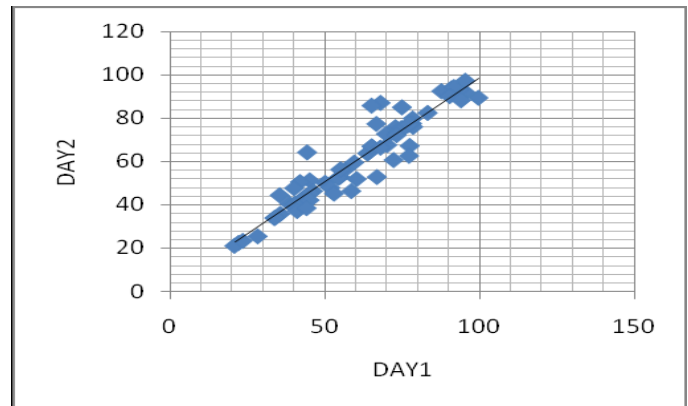

Graph 3: Females

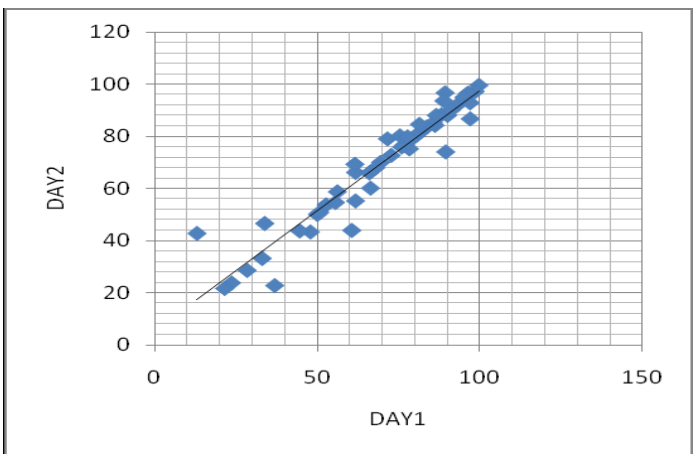

Graph 4: Males

Table 4: Correlation

\begin{tabular}{|c|c|c|c|c|c|c|c|}
\hline & $\mathrm{N}$ & Mean & SD & Mean diff & SD of diff & $\mathrm{t}$ value & $\mathrm{P}$ \\
\hline Day I & 120 & 65.30 & 21.39 & .089 & 6.607 & .148 & Ns .882 \\
Day II & 120 & 5.39 & 21.24 & .089 & & & \\
\hline
\end{tabular}

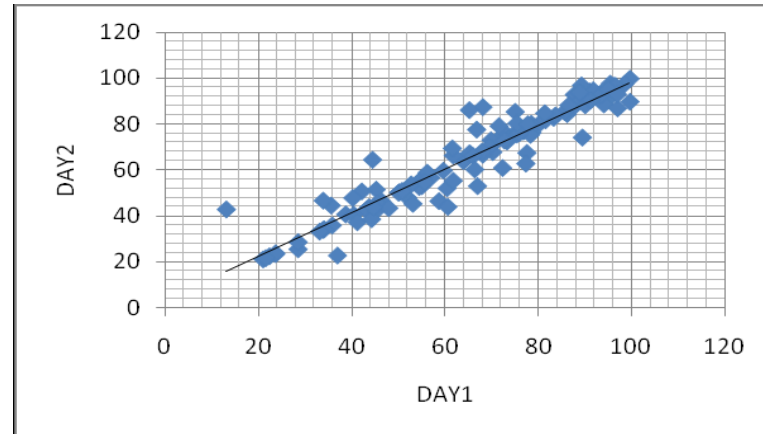

Graph 5: The correlation

Table 5: Correlation Coefficient intraclass

\begin{tabular}{|c|c|c|c|c|}
\hline Interclass & \multicolumn{2}{|c|}{$95 \%$ confidence Interval } & \multicolumn{2}{|c|}{ correlation } \\
\cline { 2 - 5 } & Lower bound & Upper bound & P value & \\
\hline .978 & .965 & .983 & .000 & Hs \\
\hline
\end{tabular}

Table 6: Interpretation of ICC

\begin{tabular}{|c|c|}
\hline \multicolumn{2}{|c|}{ Interpretation of ICC } \\
\hline$<0.40$ & Poor agreement \\
\hline $0.4-7.5$ & Fair agreement \\
\hline $0.75-0.85$ & Good agreement \\
\hline$>0.85$ & Excellent agreement \\
\hline
\end{tabular}

Musculoskeletal disorders are a serious health problem and being most important causes of disability. SPADI Questionnaires are short, easily comprehended and simple to complete. Shoulder Pain studies in the Maharashtra lacked reliable and valid assessment instruments. The aim of the study was to adapt the SPADI into Marathi language. In order to ensure quality of adaptation, this study followed the essential steps recommended by the guidelines. [11]. Independent forward and backward translators prepared the Marathi questionnaires and reviewed by a bilingual team which includes 4 translators, 1 public health physician and 3 physiotherapists, to assess the necessity and performing a culture adaptation and fine tune it for use among the Marathi knowing patients. Final stage of adaptation was performed and pre final version has been made. Initially, 10 Marathi speaking patients with shoulder pain completed the translated version. According to their remarks no revision was needed for the questionaires and final version of SPADI has been made. In this study 120 patients (females-69, males-51) of shoulder pain who fulfil the inclusion criteria were participated. The questionnaire was given twice in 24hours to measure the test-retest reliability.

This study shows the excellent reliability (ICC- .97).Our findings were higher in comparison of the other similar studies like Brazilian (ICC-.90-.94), Danish (ICC-.88), German (ICC-.94), Slovene (ICC-.94), Turkish (ICC-.92). The questionnaire demonstrated to be highly acceptable, easily understood and self administratable and no time consuming. The results of the study show that it is possible to translate functional status questionnaire into other languages without losing the psychometric properties of the original English version. 20. Researchers and clinicians may benefit from using this instrument to Marathi speaking population to measure out comes in clinical evaluation, research and treatment of patients.

\section{Conclusion}

This study has produced an approximately translated and culturally adapted Marathi version of SPADI. The Marathi version showed very good psychometric properties. As the SPADI is very for the patient to administer and score in clinical and research ,its use can be recommended among Marathi speaking patients.

\section{Future Scope}

Further studies using SPADI Marathi version are suggested to focus on minimum detectable change, minimal clinical important difference, among levels of severity as already reported for the original version.

\section{Limitations of the Study}

Criterion validity can be done in future of this scale

\section{References}

[1] Angst. F, Goldhahn.J, Pap .G, Mannion A.F, Roach K.E, Siebertz D et al. Cross-cultural adaptation, reliability and validity of the German Shoulder Pain and 


\section{International Journal of Science and Research (IJSR) \\ ISSN (Online): 2319-7064}

Index Copernicus Value (2015): 78.96 | Impact Factor (2015): 6.391

Disability Index (SPADI).Rheumatology . XXXX (3), pp.87-92, 2007.

[2] Michener LA, Snyder AR. Evaluation of health-related quality of life in patients with shoulder pain: are we doing the best we can? Clinical Sports Med. XXVII (3), pp.491-505, 2008.

[3] Martins J, Napoles B.V, Hoffman C.B, Oliveira A.S. The Brazilian version of Shoulder Pain and Disability Index - translation, cultural adaptation and reliability. Rev Bras Fisioter Sao Carlos. XIV (6), pp527-36, 2010.

[4] Roach KE, Budiman-Mak E, Songsiridej N, Lertratanakul Y. Development of a shoulder pain and disability index: Arthritis care research .IV (4), pp 1439, 1991.

[5] Breckenridge J.D: shoulder pain and disability index. Journal of Physiotherapy XVII(3) pp 197,2011.

[6] Christiansen DH, Andersen JH, Haar JP. Cross-cultural adaption and measurement properties of the Danish version of the Shoulder Pain and Disability Index. Clinical Rehabilitation. XXVII (4), pp 355-60, 2013.

[7] Sharma N , Sharma S, Kataria C. Translation and Adaptation of Shoulder Pain and Disability Index (SPADI) into Hindi-Part 1: Indian Journal of Physiotherapy and Occupational Therapy - An International Journal Year. V (4), pp 142-5, 2011.

[8] Jamnik, Helena, Spevak, Klopcic M. Shoulder Pain and Disability Index: validation of Slovene version: International Journal Of Rehabilitation Research .XXXI (4), pp 337-41, 2008.

[9] Jose A.N, Puerto-Ceballos I, Guzman-Hau W, MD, Bassol-Pereo A, Nuno-Gutierrez B.L, . Development of a Spanish Version of the shoulder disability questionnaire: Journal of Clinical Rheumatology. XI (4), pp.45, 2005.

[10]Bicer A, Ankarali H. Shoulder Pain And Disability Index: a validation study in Turkish women. Singapore Med J, XXI(11),pp865-70,2010.

[11]Beaton D et al. Guidelines for the process of crosscultural adaptation of self report measures. Spine J, XXV (24), pp.3186-3191,2000.

\section{Author Profile}

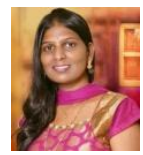

Dr. T. Poovishnudevi graduated BPT and MPT from Sri Ramakrishna College of Physiotherapy, Coimbatore. From 2009-2014 worked in swatantra College of Physiotherapy, from 2014- 2015 in Srinivas Institute of Physiotherapy and research centre and at present working as Assoc professor in Krishna college of physiotherapy, KIMSDU, Karad, Maharashtra.

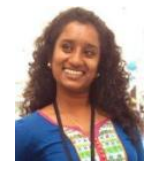

Dr. Sapna Shamarao Khot graduated BPT from Chaitanya Medical Foundation, College of Physiotherapy and MPT from Srinivas Institute Of Physiotherapy and research centre. From 2015- till date working as clinical physiotherapist in Qi Spine Clinic, Ghatkoper branch, Mumbai, Maharashtra. 